University of Nebraska - Lincoln

DigitalCommons@University of Nebraska - Lincoln

Roger Kirby Publications

Research Papers in Physics and Astronomy

April 2006

\title{
Micromagnetic energy barriers
}

\author{
Ralph Skomski \\ University of Nebraska-Lincoln, rskomski2@unl.edu \\ Jian Zhou \\ University of Nebraska-Lincoln, jzhou@unlserve.unl.edu \\ Roger D. Kirby \\ University of Nebraska-Lincoln, rkirby1@unl.edu \\ David J. Sellmyer \\ University of Nebraska-Lincoln, dsellmyer@unl.edu
}

Follow this and additional works at: https://digitalcommons.unl.edu/physics_kirby

Part of the Physics Commons

Skomski, Ralph; Zhou, Jian; Kirby, Roger D.; and Sellmyer, David J., "Micromagnetic energy barriers" (2006). Roger Kirby Publications. 3.

https://digitalcommons.unl.edu/physics_kirby/3

This Article is brought to you for free and open access by the Research Papers in Physics and Astronomy at DigitalCommons@University of Nebraska - Lincoln. It has been accepted for inclusion in Roger Kirby Publications by an authorized administrator of DigitalCommons@University of Nebraska - Lincoln. 


\title{
Micromagnetic energy barriers
}

\author{
R. Skomski, ${ }^{\text {a) }}$ J. Zhou, R. D. Kirby, and D. J. Sellmyer \\ Center for Materials Research and Analysis, University of Nebraska, Lincoln, Nebraska 68588 \\ and Department of Physics and Astronomy, University of Nebraska, Lincoln, Nebraska 68588
}

(Presented on 31 October 2005; published online 26 April 2006)

The structure of micromagnetic energy barriers responsible for slow magnetization processes is investigated. Thermally activated slow magnetization processes proceed over energy barriers whose structure is determined by the micromagnetic free energy. This restricts the range of physically meaningful energy barriers. An analysis of the underlying micromagnetic free energy yields power-law dependences with exponents of $3 / 2$ or 2 for physically reasonable models. This must be contrasted to other power laws, such as linear laws, and to $1 / \mathrm{H}$-type dependences. In the limit of small energy barriers, corrections to the Arrhenius law become important. In this regime, there is no simple expression for the relaxation behavior, but two requirements help to judge models and approximations. First, at low temperatures, the Arrhenius-type power laws must be reproduced. Second, as in the Arrhenius limit, the approaches must correspond to well-defined energy landscapes. () 2006 American Institute of Physics. [DOI: 10.1063/1.2173228]

\section{INTRODUCTION}

Thermal excitations affect the magnetic hysteresis and determine, for example, the stability of the information stored in magnetic recording media and the time dependence of the remanence of permanent magnets. A key question concerns the physical nature of the involved energy barriers $E_{a}$ over which thermal activation occurs. Various partially exclusive field dependences $E_{a}(H)$ have been proposed, and there is a continuing debate ${ }^{1-5}$ about the applicability of these expressions. One example is power laws

$$
E_{a} \sim\left(H_{\mathrm{o}}-H\right)^{m},
$$

where $H_{\mathrm{o}}$ is a switching field (or static coercivity) and the exponent $m$ depends on the considered model. Some exponents are $m=1, m=3 / 2$, and $m=2 .^{6-8}$ There have also been approaches to treat $m$ as an adjustable or field-dependent parameter, and it has been argued that $m$ implies some kind of averaging over energy barriers. Other proposed dependences are relations such as $1 / H$ and $1 / H-1 / H_{0}{ }^{9,10}$ The discussion has been fueled by the popular belief that the exponents $m$, especially $m=2$ and $m=3 / 2$, are limited to specialized or highly simplified models. ${ }^{10}$

Thermally activated magnetization reversal is usually described by the Arrhenius law

$$
\tau=\tau_{\mathrm{o}} \exp \left(\frac{E_{a}}{k_{B} T}\right),
$$

where $\tau$ is the relaxation time and $\tau_{\mathrm{o}}=1 / \Gamma_{\mathrm{o}}$ is an inverse attempt frequency of order $10^{-10} \mathrm{~s}$. This law was originally used in chemistry but has been well established in finitetemperature magnetism since the 1930s. ${ }^{11}$ Depending on the context, it is also known as the Néel or Néel-Brown relaxation law. At very low temperatures, where $\exp \left(-E_{a} / k_{B} T\right)$ is negligible, the reversal is determined by quantum tunneling, but these contributions go beyond the scope of this paper.

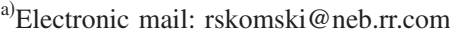

The dependence of the energy barrier $E_{a}$ on $H$ leads to a number of well-known experimental phenomena important in permanent magnets, soft magnets, and recording media. ${ }^{1-5}$ First, the remanent magnetization exhibits a logarithmic decay known as magnetic viscosity and reflecting a realstructure averaging over energy barriers. ${ }^{11}$ For example, permanent magnets lose a small fraction of their magnetization, typically a few $0.1 \%$, within the first few hours after production. ${ }^{4}$ Second, the coercivity depends on the sweep rate $d H / d t$ of the applied field. ${ }^{5}$ This effect, which can be explained as a fluctuation field, ${ }^{12}$ is related to the magnetic viscosity but occurs for both wide and narrow distributions of the activation energy.

Third, by linearizing the energy barriers, it is possible to define and measure an activation volume $V^{*} .{ }^{5,13}$ However, this experimental procedure does not mean that the energy barrier is linear, $E_{a} \sim V^{*}\left(1-H / H_{c}\right)$. In fact, most or all energy barriers are nonlinear functions of $H$, and $V^{*}$ tends to differ from the physical volume $V_{\mathrm{o}}$ of the underlying magnetization process. Note that $V_{\mathrm{o}}$ is not necessarily equal to the grain or particle volume-due to cooperative and localization effects, it may be smaller or larger than the particle volume. ${ }^{5,7}$ It is a lower bound to the Barkhausen volume $V_{B}$, because the thermally activated reversal of a small volume $V_{\mathrm{o}}$ may initiate big domain-wall jumps.

\section{ENERGY BARRIERS}

The energy barriers derive from (free) energy landscapes $E(\mathbf{M}, \mathbf{H})$ and depend-via local micromagnetic parameters such as the anisotropy $K_{1}(\mathbf{r})$. For a magnet containing $N$ atoms located at positions $\mathbf{r}_{i}$, the number of magnetic degrees of freedom is $2 N$, corresponding to the magnetization angles $\theta_{i}$ and $\phi_{i}$. In most cases, the number of relevant degrees of freedom is much smaller. For example, the pinning of a domain wall may be described by the position $x$ of the wall or, in a somewhat better approximation, by the domainwall position and the domain-wall curvature.

Figure 1 shows an energy-barrier landscape where the 


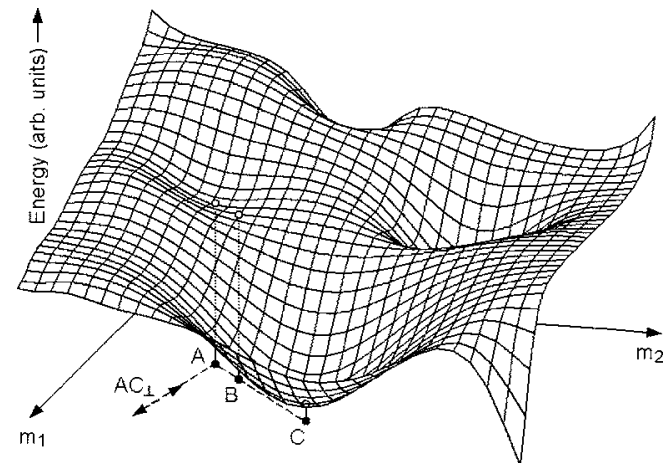

FIG. 1. Multidimensionality of the energy barriers. Only the trajectory corresponding to the lowest-lying mode contributes to the switching.

local minimum $(A)$ has a higher energy than the second local minimum $(C)$ but is separated from the latter by a saddlepoint maximum $(B)$. The states $A, B$, and $C$ are generally associated with different magnetization projections along the direction of the external field $\mathbf{H}$, so that a magnetic field can drive or support the transition. In Fig. 1, the energy barrier $E_{a}=E(B)-E(A)$. Other reversal modes, such as $A C_{\perp}$ in Fig. 1 , are not forbidden, but they involve huge energy differences $\Delta E$. Consider, for example, the reversal of a small spherical nucleus. It can be shown that any pointlike perturbation of the spin state of a magnet involves a volume of order $\delta_{B}{ }^{3}$, where $\delta_{B}$ is the domain-wall width. ${ }^{7}$ The energy $\Delta E$ of the nucleus is of order $K_{1} \delta_{B}{ }^{3}$. For Fe and Co, the temperature equivalents of this energy are $232000 \mathrm{~K}$ and $105000 \mathrm{~K}$, respectively $(100000 \mathrm{~K}=0.86 \mathrm{eV})$. The Boltzmann factor $\exp \left(-E_{a} / k_{B} T\right)$ makes giant fluctuations associated with these "arbitrary modes" 14 very unlikely. By comparison, based on an assumed waiting time of $\tau=100 \mathrm{~s}$, Eq. (1) yields the famous $25 k_{B} T$ law for the energy barriers accessible by thermal fluctuations. At room temperature, this corresponds to about $7500 \mathrm{~K}$. In practice, the external field reduces the energy barriers until they are sufficiently low $(7500 \mathrm{~K})$, as epitomized by $E(B)-E(A)$ in Fig. 1.

The relative smallness of $25 k_{B} T$ is the key to the understanding of slow magnetization dynamics. First, it requires the metastable minima to be very shallow, so that they can be described by a series expansion. ${ }^{6-8}$ Second, an analysis of the zero-temperature limit is an important tool to judge whether a proposed $E_{a}(H)$ relation is physically meaningful. Third, the path that determines $E_{a}(H)$ should correspond to a physically meaningful magnetization process. Improving agreement with experiment by treating $E_{a}(H)$ as a freely selectable function is physically meaningless and may violate basic principles of micromagnetism.

\section{POWER LAWS WITH $M=3 / 2$ AND $M=2$}

The power law of Eq. (1) was first derived by Néel, ${ }^{15}$ who obtained $m=3 / 2$. Let us consider the path $A B$ in Fig. 1 and denote the magnetization coordinate on this path as $m$. Then the energy becomes $E=E_{\mathrm{o}}+a_{1} m+a_{2} m^{2}+a_{3} m^{3}+O\left(m^{4}\right)$, where the expansion parameters $a_{1}, a_{2}$, and $a_{3}$ are welldefined functions of $M_{s}(\mathbf{r}), K_{1}(\mathbf{r})$, and $A(\mathbf{r})$. In addition, $a_{1}$ contains a projection onto the magnetic field, so that $a_{1}$ $=a_{10}+a_{1 H} H$. In equilibrium, $d E / d m=0$. This yields a qua-

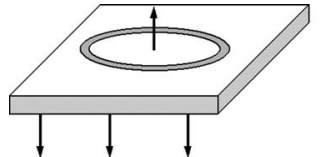

(a)

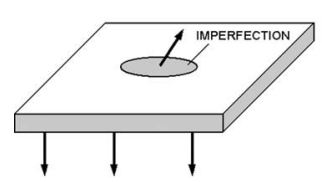

(c)

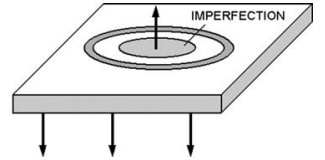

(b)

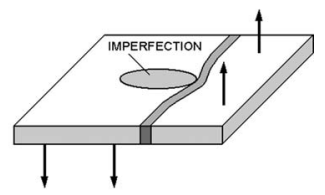

(d)
FIG. 2. Reversal modes in thin films: (a) droplet model, (b) imperfection in the center of the droplet, (c) nucleation close to an imperfection, and (d) pinning due to an imperfection. The mechanisms (c) and (d) are experimentally relevant.

dratic equation with two roots corresponding to the minimum $A$ and the saddle point $B$. The energy barrier is obtained by substituting the solutions into $E(m)$. In addition, putting $d^{2} E / d m^{2}=0$ or, alternatively, $E(A)=E(B)$, yields the static switching field $H_{\mathrm{o}}$. The result of the calculation is a power law of the type of Eq. (1) with $m=3 / 2$. When the energy barrier is symmetric, $E(-m)=-E(m)$, then $a_{3}=0$ and one must include the $m_{4}$ term. This changes the exponent to $m=2$. In other words, the exponent $m$ cannot be regarded as a fitting parameter but depends on the symmetry of the system. In most cases, $m=3 / 2,3,6,15,16$ but $m=2$ for highly symmetric systems, such as aligned Stoner-Wohlfarth particles. In particular, the $m=3 / 2$ law is realized for misaligned Stoner-Wohlfarth particles and for most domain-wall pinning mechanisms. ${ }^{7}$ Experimental values of $m$ tend to vary between 1.5 and 2.

Contrary to popular belief, ${ }^{10}$ the derivation leading to $m=3 / 2$ is very general. ${ }^{6-8}$ However, for two or more degrees of freedom, the relations $d E / d m=0$ and $d^{2} E / d m^{2}=0$ must be replaced by $\partial E / \partial m_{i}=0$ and $\partial^{2} E / \partial m_{i} \partial m_{j}=0$, respectively. ${ }^{17}$ Aside from accidental degeneracy, the directions perpendicular to the lowest-lying mode involve much higher energies and can safely be excluded.

\section{OTHER ENERGY-BARRIER EXPRESSIONS}

Several models with dependences different from Eq. (1) have been proposed using arguments from the phasetransition kinetics. ${ }^{9,10}$ The idea is outlined in Fig. 2(a). Comparing domain walls with the surface of liquid droplets of radius $R$ forming during condensation from the gas phase, the magnetic energy of a thin film is written as the sum of a Zeeman energy proportional to $H R^{2}$ and a domain-wall energy proportional to $R$. Minimizing the energy yields $1 / H$ energy barriers. However, this law amounts to the unphysical prediction of an infinite zero-field energy, and at zero temperature, the coercivity goes to infinity, in clear contrast to experiment. Subtracting a $1 / H_{\mathrm{o}}$ term, so that $E_{a} \sim 1 / H$ $-1 / H_{0},{ }^{10}$ solves a part of the problem but has no welldefined physical meaning. Scenarios such as that in Fig. 2(b) combine nucleation features with the pinning features of Fig. 2(a) but do not yield energy landscapes of the type $1 / H$ $-1 / H_{\mathrm{o}}$. In fact, formally expanding this energy into powers 


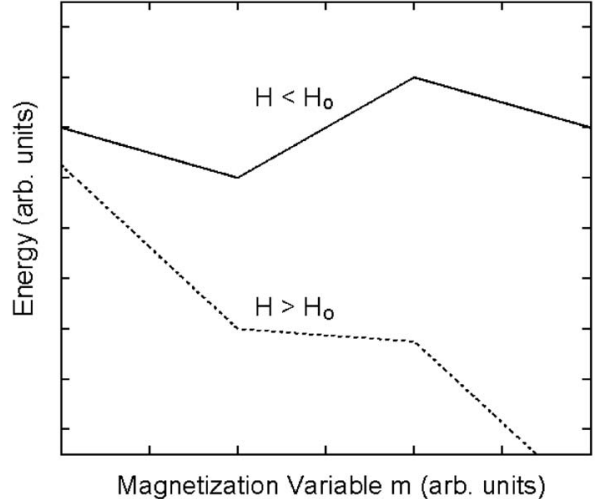

FIG. 3. Example of a fictitious energy landscape with $m=1$.

of $H-H_{\mathrm{o}}$ yields a power law with $m=1$ and should not be discussed separately from Eq. (1).

Linear laws, where $m=1$, are sometimes used in simplified models, but so far it has not been possible to derive them from physically reasonable energy landscapes. ${ }^{7,16}$ Figure 3 shows a fictitious pinning energy landscape that would yield a linear law. In reality, the singularities responsible for the (piecewise) linear nature of $E_{a}(H)$ are smoothened out by the continuous domain-wall profile.

\section{DISCUSSION AND CONCLUSIONS}

The validity of the Arrhenius law is limited to energy barriers much larger than $k_{B} T .^{17}$ In this regime, the above equations provide a comprehensive and self-consistent description. In the superparamagnetic limit, this may no longer be true, because the expansion in Sec. III requires the energy barriers to vary smoothly on a scale of order of $25 k_{B} T$. When the energy landscape contains "hills" whose size is comparable to or smaller than $25 k_{B} T$, then higher-order terms must be included and the deviations from the $m=3 / 2$ and $m=2$ power laws are possible. This includes small nanoparticles, thin films, and thin nanowires where $V_{\mathrm{o}}$ is very small due to particle size, film thickness, or wire diameter. ${ }^{18}$ An example is spins captured in a deep valley with steep slopes, where the Zeeman energy $-\mu_{\mathrm{o}} \mathbf{M} \cdot \mathbf{H} V_{\mathrm{o}}$ leads to an asymptotic exponent $m=1$ that may be thermally accessible for very small feature volumes $V_{0}$. However, as discussed in,${ }^{14}$ this does not mean that the $m=3 / 2$ and $m=2$ predictions can be replaced by "arbitrary" models which describe certain time, field, or temperature windows but crudely misinterpret the nature of the magnetization reversal. Any meaningful model must be related to the real structure of the magnet, and the underlying parameters such as interatomic exchange and anisotropy must be compatible with the magnetism of the investigated system.

In the limit of very fast processes, features such as Landau-Lifshitz damping and precession interfere. First, a Fokker-Planck analysis ${ }^{18}$ reveals that the applicability of the Arrhenius-Becker-Kramer or Néel-Brown law $\exp \left(-E / k_{B} T\right)$ for the relaxation rate is no longer ensured. Figure 4 illustrates this point by considering an energy landscape with multiple saddle points. At high temperatures, the number of accessible paths increases without extra expense in energy. Second, the dependence $E_{a}(H)$ is likely to be more compli-

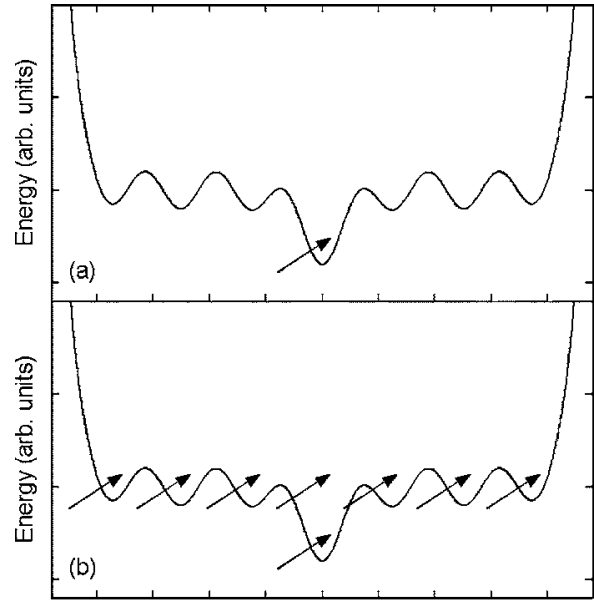

Horizontal Spin Coordinate (arb. units)

FIG. 4. Activation entropy: (a) low temperature and (b) high temperature. In this example, the transition rate $\Gamma_{o}$ changes to about $7 \Gamma_{o}$.

cated than Eq. (1). Third, the energy landscape exhibits an explicit temperature dependence via $K_{1}(T)$, and in small particles, thermodynamic fluctuations $\left\langle K_{1}^{2}\right\rangle-\left\langle K_{1}\right\rangle^{2}$ may be important.

In summary, we have shown that the energy barriers responsible for thermally activated slow magnetization dynamics are of the power-law type, with exponents $m=3 / 2$ or 2 , depending on the symmetry of the problem. In contrast to popular belief, these laws are not restricted to aligned StonerWohlfarth particles but also describe a broad range of pinning and nucleation mechanisms. Other expressions may reproduce some features of the magnetic behavior but tend to violate other criteria, such as a meaningful low-temperature limit.

\section{ACKNOWLEDGMENTS}

This research is supported by NSF-MRSEC, the W. M. Keck Foundation, INSIC, and CMRA.

${ }^{1}$ P. Gaunt, Philos. Mag. 34, 775 (1976).

${ }^{2}$ E. P. Wohlfarth, J. Phys. F: Met. Phys. 14, L155 (1984).

${ }^{3}$ P. Gaunt, J. Appl. Phys. 59, 4129 (1986).

${ }^{4}$ D. Givord and M. F. Rossignol, in Rare-Earth Iron Permanent Magnets, edited by J. M. D. Coey (Oxford University Press, Oxford, 1996), p. 218. ${ }^{5}$ D. J. Sellmyer, M. Yu, R. A. Thomas, Y. Liu, and R. D. Kirby, Physics of Low-Dimensional Semiconductor Structures, Special Issue 1-2, 155 (1998).

${ }^{6}$ R. H. Victora, Phys. Rev. Lett. 63, 457 (1989).

${ }^{7}$ R. Skomski, J. Phys.: Condens. Matter 15, R841 (2003).

${ }^{8}$ R. Skomski, D. Leslie-Pelecky, R. D. Kirby, A. Kashyap, and D. J. Sellmyer, Scr. Mater. 48, 857 (2003).

${ }^{9}$ T. Egami, Phys. Status Solidi A 20, 157 (1973); Phys. Status Solidi B 57, 211 (1973)

${ }^{10}$ J. Moritz, B. Dieny, J. P. Nozières, Y. Pennec, J. Camarero, and S. Pizzini, Phys. Rev. B 71, 100402 (2005).

${ }^{11}$ R. Becker and W. Döring, Ferromagnetismus (Springer, Berlin, 1939).

${ }^{12}$ L. Néel, J. Phys. Radium 12, 339 (1951).

${ }^{13}$ R. Street and J. C. Wooley, Proc. Phys. Soc., London, Sect. A 62, 562 (1949).

${ }^{14} \mathrm{~A}$. Aharoni, Introduction to the Theory of Ferromagnetism (Oxford University Press, Oxford, 1996).

${ }^{15}$ L. Néel, J. Phys. Radium 11, 49 (1950).

${ }^{16}$ R. Skomski and J. M. D. Coey, Permanent Magnetism (IOP, Bristol 1999).

${ }^{17}$ R. D. Kirby, M. Yu, and D. J. Sellmyer, J. Appl. Phys. 87, 5696 (2000).

${ }^{18}$ H. A. Kramers, Physica (Amsterdam) 7, 284 (1940). 